\title{
A high triglyceride glucose index is more closely associated with hypertension than lipid or glycemic parameters in elderly individuals: a cross-sectional survey from the Reaction Study
}

Binruo Zhu ${ }^{1,2+}$, Jie Wang ${ }^{1,2+}$, Kang Chen², Wenhua Yan², Anping Wang², Weiqing Wang ${ }^{3}$, Zhengnan Gao ${ }^{4}$, Xulei Tang ${ }^{5}$, Li Yan', Qin Wan' ${ }^{7}$ Zuojie Luo ${ }^{8}$, Guijun Qin ${ }^{9}$, Lulu Chen ${ }^{10}$ and Yiming Mu ${ }^{1,2^{*}}$ (B)

\begin{abstract}
Background: Both lipid and glucose abnormalities are associated with hypertension (HTN). However, it is unclear whether the triglyceride-glucose (TyG) index is associated with HTN. Therefore the aim of this study is to investigate the association of the TyG index and HTN and to compare the discriminative power of the TyG index, lipid, glycemic parameters for the risk of HTN in elderly individuals.

Methods: The present study was nested in a longitudinal (REACTION) study from May 2011 to December 2011, which was designed to demonstrate the association of abnormal glucose metabolism with the risk of cancer in the Chinese population. In total, 47,808 participants were recruited in this cross-sectional study. The TyG index was divided into five groups: the <20\% group, the 20-39\% group, the 40-59\% group, the 60-79\% group and the $\geq 80 \%$ group, according to quintile division of the subjects. Three multivariate logistic regression models were used to evaluate the association between the TyG vs. lipid parameters, glycemic parameters and HTN.

Results: Multivariate logistic regression analysis shows that compared with lipid and glycemic parameters, the TyG index remains significantly associated with HTN in either total subjects or subjects separated into men and women (odds ratio (OR) 1.33, 95\% confidence interval (Cl) 1.18-1.51, $\mathrm{p}<0.0001$ in total subjects; OR 1.39, 95\% Cl 1.11-1.74, $\mathrm{p}=0.0042$ in men; OR $1.28,95 \% \mathrm{Cl} 1.11-1.49, \mathrm{p}=0.0010 \mathrm{in}$ women). In a stratified analysis, an elevated TyG index is significantly associated with HTN in the subgroup of the oldest age ( $\geq 65)(\mathrm{OR} 1.67,95 \% \mathrm{Cl} 1.30-2.14, \mathrm{p}<0.0001)$, as well as with obesity (Body mass index (BMI) $\left.\geq 28 \mathrm{~kg} / \mathrm{m}^{2}\right)(\mathrm{OR} 1.85,95 \% \mathrm{Cl} 1.29-2.66, \mathrm{p}=0.0009)$ or lower estimated glomerular filtration rate (eGFR) $\left(<90 \mathrm{~mL} /\left(\min \cdot 1.73 \mathrm{~m}^{2}\right)\right)(\mathrm{OR} 1.72,95 \% \mathrm{Cl} 1.33-2.21, \mathrm{p}<0.0001)$.

Conclusion: The TyG index is significantly associated with HTN and shows the superior discriminative ability for HTN compared with lipid and glycemic parameters in the Chinese elderly population.
\end{abstract}

Keywords: TyG index, HTN, Lipid parameters, Glycemic parameters

*Correspondence: muyiming@301hospital.com.cn

${ }^{\dagger}$ Binruo Zhu and Jie Wang contributed equally to this work

${ }^{2}$ Department of Endocrinology, Chinese PLA General Hospital, 28 Fu Xing Road, Beijing 100853, People's Republic of China

Full list of author information is available at the end of the article

\section{Background}

Hypertension (HTN) is one of the most prevalent cardiovascular risk factors, with over $34 \%$ of males and $28 \%$ of women aged $\geq 25$ years being affected globally by raised

(c) The Author(s) 2020. This article is licensed under a Creative Commons Attribution 4.0 International License, which permits use, sharing, adaptation, distribution and reproduction in any medium or format, as long as you give appropriate credit to the original author(s) and the source, provide a link to the Creative Commons licence, and indicate if changes were made. The images or other third party material in this article are included in the article's Creative Commons licence, unless indicated otherwise in a credit line to the material. If material is not included in the article's Creative Commons licence and your intended use is not permitted by statutory regulation or exceeds the permitted use, you will need to obtain permission directly from the copyright holder. To view a copy of this licence, visit http://creativeco mmons.org/licenses/by/4.0/. The Creative Commons Public Domain Dedication waiver (http://creativecommons.org/publicdomain/ zero/1.0/) applies to the data made available in this article, unless otherwise stated in a credit line to the data. 
blood pressure [1]. With $20 \%$ of the world's population, China represents a large portion of this burden, where HTN and blood pressure-related cardiovascular diseases (CVDs) are major public health challenges [2, 3]. HTN prevalence has risen in recent decades, resulting in an increase of blood pressure-related morbidity and mortality.

It is well known that both lipid and glucose abnormalities are associated with HTN. It is reported that dyslipidemia has been observed in $50 \%$ to $80 \%$ of hypertensive patients [4]. Dyslipidemia, comprising elevated triglyceride (TG), high cholesterol (TC), increased low-density lipoprotein cholesterol (LDL-C), and decreased highdensity lipoprotein cholesterol (HDL-C), is independently associated with HTN or other CVDs risk factors [5-8]. There are renewed interests engendered by epidemiological and genetic evidence proving that increased TG, remnant TC, or TG-rich lipoproteins are additional causes of CVDs and all-cause mortality [9]. Similarly, HTN and type 2 diabetes (T2DM) are common causes of morbidity; both constitute risk factors for CVDs and might be engaged in similar genetic and environmental risk factors [10]. It is reported that elevated plasma glucose is a steady and independent predictor of HTN [11]. Some modern antidiabetic drugs are also capable of lowering both office and ambulatory blood pressure. This can contribute to the favorable effect on some clinical endpoints, most importantly the reduction of congestive heart failure and cardiovascular mortality $[12,13]$.

The main pathogenetic pathways linking T2DM, dyslipidemia and HTN are thought to be through insulin resistance (IR) and increased activity of the sympathetic nervous system and the renin-angiotensin-aldosterone system as well as increased renal sodium reabsorption [14]. The association between IR and the risk of incident HTN was shown in a recent meta-analysis of 11 studies [15], suggesting that IR could be employed as an adjunctive tool to identify individuals at potential risk for HTN. Glucose clamp technique is the gold standard for IR measurement initially proposed by De Fronzo [16]. However, such direct diagnostic tests have considerably high costs and low availability for epidemiologic use [17].

In recent years, the triglyceride-glucose (TyG) index is arising as an ideal substitution for IR [18, 19]. It is calculated as $\ln$ [fasting plasma glucose $(\mathrm{FBG})(\mathrm{mg} / \mathrm{dL})$ * TG (mg/dL)/2] [20]. This measurement merely requires simple lab tests like TG and plasma glucose, which can be obtained in highly cost-effective and time-efficient ways. Additionally, the TyG index has been revealed to determine IR in a more appropriate way than other substitutional indexes like HOMA-IR, which was compared with the gold standard method for IR [21]. Previous studies showed that the TyG index is closely associated with
HTN [22], artery stiffness and coronary artery calcification [23-25]. Furthermore, the TyG index can predict coronary artery disease severity and cardiovascular outcomes [26]. The association between the TyG index and T2DM was also demonstrated in Spain, China and Korea [19, 27-29].

However, studies are limited that involve the association between the TyG index and HTN and comparison of the discriminative abilities of the TyG index, lipid, glycemic parameters for the risk of HTN. Therefore our study intends to explore the association of the TyG index with HTN and compare the discriminative power of the TyG index, lipid, glycemic parameters for HTN in elderly individuals in China.

\section{Methods}

\section{Study subjects}

The present study assessed 47,808 participants aged over 40 years from a longitudinal REACTION study (Risk Evaluation of cAncers in Chinese diabeTic Individuals), including seven regional centers (Gansu, Guangdong, Henan, Hubei, Liaoning, Shanghai, and Sichuan), from May 2011 to December 2011. Previous history of related chronic diseases, using ACEI/ARB medicines, lipid-lowering drugs, missed data and/or included outliers, were exclusion criteria in the study. Finally, 43,591 participants were recruited (Fig. 1).

Before the investigation, the clinicians were welltrained for the questionnaire and data collection. The present study was approved by the Committee on Human Research at Rui-Jin Hospital affiliated with the School of Medicine, Shanghai Jiao Tong University and all participants recruited had signed informed consents before data collection.

\section{Clinical data and biochemical indicators}

The subjects received the following examinations: a standardized questionnaire, anthropometric measurements, blood collection, and a standard 75-goral glucose tolerance test (OGTT) or standard meal test. The same trained clinicians carried out standard questionnaires, which included demography, lifestyle, history of diabetes, stroke, coronary heart disease (CHD), HTN and dyslipidemia as well as the medication history, including the use of drugs. All data were maintained corresponding to established standard methods by the same well-trained clinicians. Physical examination items included height, weight, waist circumference, hip circumference, blood pressure, and heart rate. Height was measured in bare feet accurate to $0.01 \mathrm{~m}$. Weight was measured in light clothes accurate to $0.1 \mathrm{~kg}$. Waist circumference and hip circumference were measured to an accuracy of $0.01 \mathrm{~m}$ by the same staff. Waist circumference/hip circumference 
Participants from 7 centers of Reaction study: Liaoning 10140, Gansu 10026, Guangzhou 9743, Sichuan 8105,

Shanghai 6821, Henan1978, Hubei 995

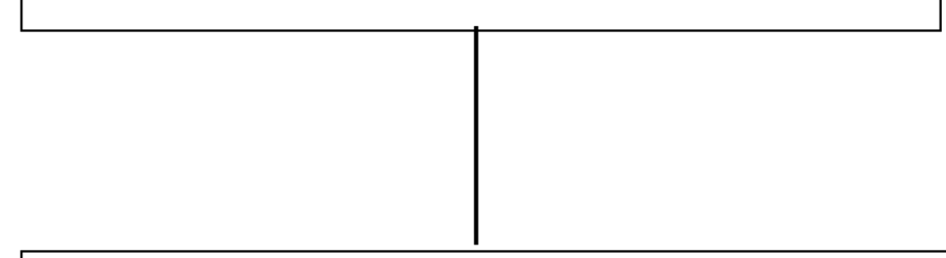

A total of 47808 participats aged over 40 years were recruted

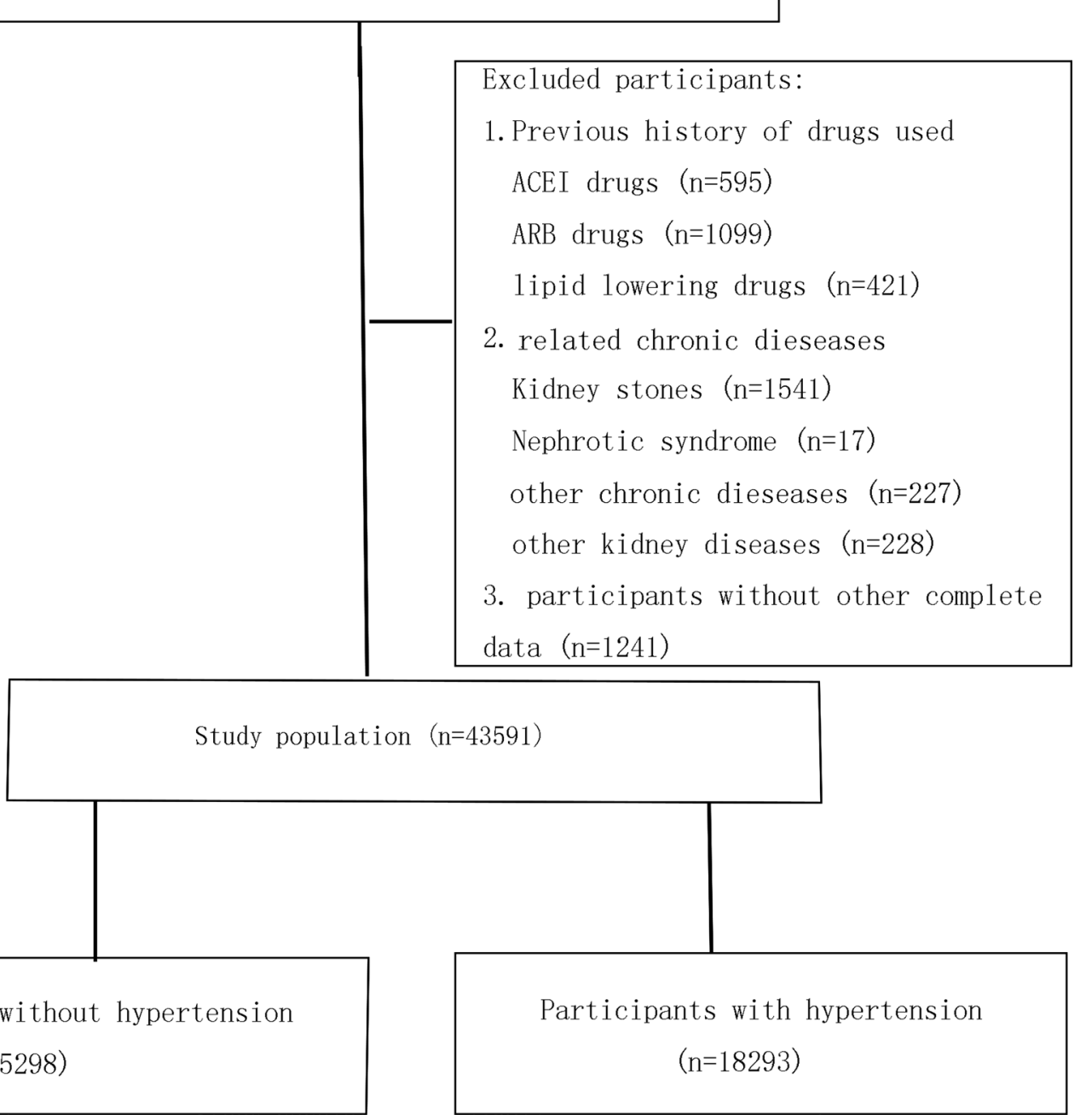

Fig. 1 Flow chart of the selection of study participants

(WHR) was accurate to 0.01. Body mass index (BMI) was calculated as weight/height ${ }^{2}$. After at least a 5-minute rest, blood pressure was measured seated three times with an interim of $1 \mathrm{~min}$, using an OMRON electronic blood pressure monitor. The average blood pressure was calculated and used for analysis. The estimated 
glomerular filtration rate (eGFR) was determined by the modified MDRD equation [30]: eGFR $=186 \times($ serum creatinine $\times 0.011) \quad-1.154 \times(\text { age })^{-0.203} \times(0.742$ if female $) \times 1.233$. TyG $(\mathrm{mg} / \mathrm{dl})^{2}=\ln [$ FBG $(\mathrm{mg} / \mathrm{dl}) *$ TG $(\mathrm{mg} / \mathrm{dl}) / 2]$.

\section{$75 \mathrm{~g}$ OGTT or standard meal test}

After an overnight fast for at least $12 \mathrm{~h}$, the first fasting blood samples were obtained for FBG measurement. Standard $75 \mathrm{~g}$ glucose solution was given to the individuals without a T2DM history, while standard meals containing $100 \mathrm{~g}$ carbohydrates were given to the individuals with a T2DM history. Blood samples for glucose measurement were obtained at 120 min after either $75 \mathrm{~g}$ OGTT or standard meal test. FBG and $2 \mathrm{~h}$ post-load blood glucose (PBG) were measured by the Hexokinase method on an autoanalyzer.

TG, TC, HDL-C, LDL-C, alanine transferase (ALT), aspartate transferase (AST), serum creatinine (Cr) and gamma-glutamyl transferase (GGT) were measured by chemiluminescence on an autoanalyzer. Glycosylated hemoglobin (HbA1c) was measured by high pressure liquid chromatography.

\section{Definition of variables}

TyG index was divided into five groups: the $<20 \%$ group, the $20-39 \%$ group, the $40-59 \%$ group, the $60-79 \%$ group and the $\geq 80 \%$ group, according to quintile division of the subjects. As the Chinese guideline for the management of dyslipidemia in adults (revised in 2016) [31] suggests, lipid parameters were categorized as follows: 1 . TG: normal: $<1.7 \mathrm{mmol} / \mathrm{L}$, borderline high: $1.7-2.3 \mathrm{mmol} / \mathrm{L}$, high: $\geq 2.3 \mathrm{mmol} / \mathrm{L} ; 2$. TC: normal: $<5.2 \mathrm{mmol} / \mathrm{L}$, borderline high: $5.2-6.2 \mathrm{mmol} / \mathrm{L}$, high: $\geq 6.2 \mathrm{mmol} / \mathrm{L} ; 3$. HDL-C: low risk: $\geq 1.0 \mathrm{mmol} / \mathrm{L}$, high risk: $<1.0 \mathrm{mmol} / \mathrm{L} ; 4$. LDL-C: ideal $<2.6 \mathrm{mmol} / \mathrm{L}$, borderline high: $3.4-4.1 \mathrm{mmol} /$, high: $\geq 4.1 \mathrm{mmol} / \mathrm{L}$. According to the WHO guidelines, T2DM was defined as $\mathrm{FBG} \geq 7.0 \mathrm{mmol} / \mathrm{L}$, or $\mathrm{PBG} \geq 11.1 \mathrm{mmol} / \mathrm{L}$, or a selfreported history of T2DM. HbA1c was divided into two groups according to the American Diabetes Association [32] and the World Health Organization [33]: normal: $<6.5 \%$ and high $\geq 6.5 \%$. HTN was defined as systolic blood pressure (SBP) $\geq 140 \mathrm{mmHg}$ or diastolic blood pressure (DBP) $\geq 90 \mathrm{mmHg}$, or diagnosed as hypertension by clinicians and meanwhile undergoing antihypertensive-medication therapy. The subjects who were once diagnosed as hypertension but present status could not meet diagnostic criteria were treated as normotensive in present study. Participants were divided into three groups according to their smoking frequency: no: never or have already quit smoking; occasional: smoking less than once a week or less than 7 cigarettes weekly; frequently: smoking one or more cigarettes daily for at least a half year. Similarly, participants were divided into three groups according to their alcohol intake frequency: no: never or have already quit drinking; occasional: drinking less than once a week; frequently: drinking more than once a week for at least a half year. Stroke, including all subtypes, was determined according to a subject's self-report, including a history of language or physical dysfunction lasting over $24 \mathrm{~h}$ and ischemic or hemorrhagic stroke by imageological diagnosis. CHD events were defined as any self-report history of hospital-admitted myocardial infarction or angina, or coronary revascularization. CVDs were also screened according to a subject's selfreport, including history of CHD, stroke, or myocardial infarction.

\section{Statistical analysis}

Empower(R) (www.empowerstats.com, X\&Y Solutions Inc., Boston, MA) and R (http://www.Rproject.org) were employed to perform the statistical analyses. The odds ratio (OR) and the $95 \%$ confidence intervals (CI) were calculated. $\mathrm{P}$ values $<0.05$ (2-sided) were considered to indicate statistical significance.

Variables were presented as mean \pm standard deviation (SD), if normal distribution. And if they were not, they were presented as median (Q1-Q3), or $\mathrm{n}(\%)$. The differences between continuous variables were compared using the Kruskal-Wallis test. The percentage difference between groups was compared using the $x^{2}$ test. Three multivariate logistic regression models were built to identify the associations between TyG vs. lipid parameters, PBG, HbA1c and HTN. Model 0 was not adjusted for any confounding factors, while Model 1 was adjusted for age and sex. Model 2 was further adjusted for age; center; sex; history of CVDs; history of T2DM; hypoglycemic drugs; SBP; DBP; BMI; ALT; AST; WHR; eGFR; smoking habits and drinking habits. Stratified analyses were conducted by the different level of age (G1: $<55$, G2: 55-65, G3: $\geq 65$ ), BMI (underweight: $<18.5 \mathrm{~kg} / \mathrm{m}^{2}$; normal weight: $18.5-24 \mathrm{~kg} / \mathrm{m}^{2}$; overweight: $24-28 \mathrm{~kg} / \mathrm{m}^{2}$, obesity: BMI of $\geq 28 \mathrm{~kg} / \mathrm{m}^{2}$, according to Cooperative Meta-analysis Group of China Obesity Task Force report [34]) and eGFR (G1: $<90 \mathrm{~mL} /$ $\left(\min \cdot 1.73 \mathrm{~m}^{2}\right), \mathrm{G} 2: \geq 90 \mathrm{~mL} /\left(\min \cdot 1.73 \mathrm{~m}^{2}\right)$, according to KDIGO [35]). Subjects were stratified into subgroups to separately explore the relevant underlying factors which might affect the relationship between the TyG index and HTN. Meanwhile, potential interactions of 
the TyG index and strata variables were assessed in the logistical regression analysis.

\section{Results}

\section{Characteristics of study population by HTN}

The study included 43,591 participants (Table 1), of whom 18,293 (40.5\%) had HTN. Compared to nonhypertensive participants, the hypertensive ones were older, possessed a larger BMI, were less frequent smokers, less frequent drinkers, had a higher mean SBP and DBP, a higher heart rate, a less favorable metabolic profile (FBG, PBG, HbA1c, AST, ALT, GGT, TC, TG), lower levels of eGFR, and a higher frequency of CVDs and T2DM.

\section{Association of the TyG index, glycemic, lipid parameters with HTN}

Multiple logistic regression models that consider separately each index and their individual components as

Table 1 Characteristics of study population by HTN

\begin{tabular}{|c|c|c|c|}
\hline & No HTN & HTN & $P$ value \\
\hline N & 25,298 & 18,293 & \\
\hline Age, years & $55.41(50.05-60.73)$ & $60.89(55.38-68.15)$ & $<0.001$ \\
\hline $\mathrm{BMI}, \mathrm{kg} / \mathrm{m}^{2}$ & $23.46(21.47-25.67)$ & $25.33(23.23-27.64)$ & $<0.001$ \\
\hline $\mathrm{SBP}, \mathrm{mmHg}$ & $121.00(112.00-130.00)$ & $149.00(140.00-162.00)$ & $<0.001$ \\
\hline $\mathrm{DBP}, \mathrm{mmHg}$ & $73.00(68.00-79.00)$ & $85.00(77.00-92.00)$ & $<0.001$ \\
\hline $\mathrm{HR}$ & $78.00(71.00-86.00)$ & $79.00(72.00-88.00)$ & $<0.001$ \\
\hline $\mathrm{FBG}, \mathrm{mmol} / \mathrm{L}$ & $5.40(5.02-5.91)$ & $5.77(5.29-6.58)$ & $<0.001$ \\
\hline $\mathrm{PBG}, \mathrm{mmol} / \mathrm{L}$ & $7.00(5.81-8.84)$ & $8.29(6.60-11.24)$ & $<0.001$ \\
\hline HbA1c, \% & $5.80(5.60-6.20)$ & $6.00(5.70-6.50)$ & $<0.001$ \\
\hline$A L T, U / L$ & $14.00(10.00-20.00)$ & $16.00(12.00-22.00)$ & $<0.001$ \\
\hline AST, U/L & $20.00(16.00-24.00)$ & $21.00(17.00-25.00)$ & $<0.001$ \\
\hline GGT, U/L & $19.00(14.00-28.00)$ & $23.00(16.00-35.00)$ & $<0.001$ \\
\hline $\mathrm{TG}, \mathrm{mmol} / \mathrm{L}$ & $1.24(0.90-1.79)$ & $1.52(1.08-2.17)$ & $<0.001$ \\
\hline $\mathrm{TC}, \mathrm{mmol} / \mathrm{L}$ & $4.93(4.21-5.68)$ & $5.14(4.38-5.88)$ & $<0.001$ \\
\hline LDL-C, mmol/L & $2.84(2.28-3.45)$ & $2.99(2.40-3.61)$ & $<0.001$ \\
\hline $\mathrm{HDL}-\mathrm{C}, \mathrm{mmol} / \mathrm{L}$ & $1.31(1.09-1.55)$ & $1.25(1.06-1.47)$ & $<0.001$ \\
\hline $\mathrm{eGFR}, \mathrm{mL} /\left(\min \cdot 1.73 \mathrm{~m}^{2}\right)$ & 96.85 (93.10-100.56) & $92.98(88.31-96.85)$ & $<0.001$ \\
\hline Sex, $(\%)$ & & & $<0.001$ \\
\hline Male & $6918(27.35 \%)$ & 6201 (33.90\%) & \\
\hline Female & $18,380(72.65 \%)$ & $12,092(66.10 \%)$ & \\
\hline Drinking, (\%) & & & $<0.001$ \\
\hline Never & $18,643(73.69 \%)$ & $14,023(76.66 \%)$ & \\
\hline Occasional & $5278(20.86 \%)$ & $2883(15.76 \%)$ & \\
\hline Frequently & 1377 (5.44\%) & 1387 (7.58\%) & \\
\hline Smoking, (\%) & & & $<0.001$ \\
\hline Never & $21,443(84.76 \%)$ & $15,779(86.26 \%)$ & \\
\hline Occasional & 862 (3.41\%) & 495 (2.71\%) & \\
\hline Frequently & 2993 (11.83\%) & 2019 (11.04\%) & \\
\hline CVDs, (\%) & & & $<0.001$ \\
\hline Yes & 681 (2.69\%) & 1690 (9.24\%) & \\
\hline No & 24,617 (97.31\%) & $16,603(90.76 \%)$ & \\
\hline T2DM, (\%) & & & $<0.001$ \\
\hline No & 22,723 (89.82\%) & 14,226 (77.77\%) & \\
\hline Yes & 2575 (10.18\%) & 4067 (22.23\%) & \\
\hline
\end{tabular}

Data were mean $\pm S D$ or median (IQR) for skewed variables or numbers (proportions) for categorical variables

$H T N$ hypertension, $B M I$ body mass index, SBP systolic blood pressure, DBP diastolic blood pressure, $H R$ heart rate, $F B G$ fasting plasma glucose, $P B G 2 \mathrm{~h}$ post-load blood glucose, $H b A 1$ c glycosylated hemoglobin, ALT alanine transferase, AST aspartate transferase, GGT gamma-glutamyl transferase, TG triglyceride, TC high cholesterol, LDL-C low-density lipoprotein cholesterol, HDL-C high-density lipoprotein cholesterol, eGFR estimated glomerular filtration rate, CVD cardiovascular disease, $T 2 D M$ type 2 diabetes 
Table 2 Association of the TyG index, glycemic, lipid parameters with HTN in total subjects

\begin{tabular}{|c|c|c|c|c|c|c|}
\hline \multirow[t]{2}{*}{ Variable } & \multicolumn{2}{|l|}{ Non-adjusted } & \multicolumn{2}{|l|}{ Adjust I } & \multicolumn{2}{|l|}{ Adjust II } \\
\hline & OR $(95 \% \mathrm{Cl})$ & P-value & OR $(95 \% \mathrm{Cl})$ & P-value & OR $(95 \% \mathrm{Cl})$ & P-value \\
\hline \multicolumn{7}{|l|}{$\mathrm{TG}, \mathrm{mmol} / \mathrm{L}$} \\
\hline$<1.7$ & 1.0 & & 1.0 & & 1.0 & \\
\hline$\geq 1.7,<2.3$ & $1.72(1.63,1.81)$ & $<0.0001$ & $1.63(1.55,1.72)$ & $<0.0001$ & $1.25(1.13,1.38)$ & $<0.0001$ \\
\hline$\geq 2.3$ & $1.99(1.89,2.10)$ & $<0.0001$ & $2.01(1.91,2.12)$ & $<0.0001$ & $1.19(1.07,1.31)$ & 0.0007 \\
\hline \multicolumn{7}{|l|}{$\mathrm{TC}, \mathrm{mmol} / \mathrm{L}$} \\
\hline$<5.2$ & 1.0 & & 1.0 & & 1.0 & \\
\hline$\geq 5.2,<6.2$ & $1.28(1.23,1.34)$ & $<0.0001$ & $1.25(1.19,1.31)$ & $<0.0001$ & $0.94(0.86,1.02)$ & 0.1432 \\
\hline$\geq 6.2$ & $1.46(1.39,1.55)$ & $<0.0001$ & $1.39(1.31,1.48)$ & $<0.0001$ & $0.90(0.81,1.01)$ & 0.0620 \\
\hline \multicolumn{7}{|c|}{$\mathrm{HDL}-\mathrm{C}, \mathrm{mmol} / \mathrm{L}$} \\
\hline$\geq 1$ & 1.0 & & 1.0 & & 1.0 & \\
\hline$<1$ & $1.18(1.13,1.25)$ & $<0.0001$ & $1.15(1.09,1.22)$ & $<0.0001$ & $1.28(1.15,1.42)$ & $<0.0001$ \\
\hline \multicolumn{7}{|c|}{ LDL-C, mmol/L } \\
\hline$<3.4$ & 1.0 & & 1.0 & & 1.0 & \\
\hline$\geq 3.4,<4.1$ & $1.31(1.25,1.37)$ & $<0.0001$ & $1.24(1.18,1.31)$ & $<0.0001$ & $1.00(0.91,1.09)$ & 0.9455 \\
\hline$\geq 4.1$ & $1.48(1.39,1.57)$ & $<0.0001$ & $1.39(1.30,1.48)$ & $<0.0001$ & $1.02(0.90,1.15)$ & 0.7711 \\
\hline \multicolumn{7}{|c|}{ TYG in transform Q5 } \\
\hline Q1 & 1.0 & & 1.0 & & 1.0 & \\
\hline Q2 & $1.54(1.44,1.64)$ & $<0.0001$ & $1.41(1.31,1.51)$ & $<0.0001$ & $1.06(0.94,1.20)$ & 0.3362 \\
\hline Q3 & $2.02(1.89,2.15)$ & $<0.0001$ & $1.76(1.64,1.88)$ & $<0.0001$ & $1.10(0.98,1.24)$ & 0.1173 \\
\hline Q4 & $2.80(2.62,2.98)$ & $<0.0001$ & $2.36(2.21,2.53)$ & $<0.0001$ & $1.33(1.18,1.51)$ & $<0.0001$ \\
\hline Q5 & $3.79(3.55,4.04)$ & $<0.0001$ & $3.32(3.10,3.55)$ & $<0.0001$ & $1.26(1.11,1.44)$ & 0.0005 \\
\hline \multicolumn{7}{|l|}{$\mathrm{HbA} 1 \mathrm{c}, \%$} \\
\hline$<6.5$ & 1.0 & & 1.0 & & 1.0 & \\
\hline$\geq 6.5$ & $2.19(2.08,2.30)$ & $<0.0001$ & $1.76(1.67,1.85)$ & $<0.0001$ & $0.96(0.84,1.10)$ & 0.5782 \\
\hline \multicolumn{7}{|l|}{ PBG, mmol/L } \\
\hline$<11.1$ & 1.0 & & 1.0 & & 1.0 & \\
\hline$\geq 11.1$ & $2.40(2.28,2.52)$ & $<0.0001$ & $1.94(1.84,2.04)$ & $<0.0001$ & $1.11(0.96,1.27)$ & 0.1535 \\
\hline
\end{tabular}

Model 0: Adjusted for no confounding factors

Model 1: Adjusted for age and gender

Model 2: age; center; sex; history of CVDs; history of T2DM; hypoglycemic drugs; SBP; DBP; BMI; ALT; AST; WHR; eGFR; smoking habits; drinking habits

predictors of HTN were constructed. Table 2 shows OR and $95 \% \mathrm{CI}$ of HTN with the groups of TG, TC, HDL-C, LDL-C, TyG quintiles, HbA1c and PBG in the total population within three different models. As seen in Table 2, every index is significantly associated with HTN in the non-adjusted model. However, after further adjustments in Model II, only the fourth and fifth quintiles of TyG (fourth quintile: OR 1.33, 95\% CI 1.18-1.51, p <0.0001; fifth quintile: OR $1.26,95 \%$ CI $1.11-1.44, \mathrm{p}=0.0005)$, HDL-C $\geq 1 \quad \mathrm{mmol} / \mathrm{L} \quad(\mathrm{OR} \quad 1.28,95 \%$ CI $1.15-1.42$, $\mathrm{P}=0.0005), \quad \mathrm{TG} \geq 1.7 \mathrm{mmol} / \mathrm{L} \quad(1.7 \leq \mathrm{TG}<2.3 \mathrm{mmol} / \mathrm{L}$ : OR $1.25,95 \%$ CI $1.13-1.38, \mathrm{P}<0.0001 ; \mathrm{TG} \geq 2.3 \mathrm{mmol} / \mathrm{L}$ : OR 1.19, 95\% CI 1.07-1.31, $\mathrm{P}=0.0007$ ) and PBG (OR 1.02 , 95\% CI $1.00-1.03, \mathrm{p}=0.0117)$ remained significantly associated with HTN, whereas TC, HDL-C, LDL$\mathrm{C}, \mathrm{HbA1c}$ were not. The above results are displayed as a color-coded figure shown in Fig. 2. The association between hypertension and continuous values of the TyG index, glycemic, lipid parameters is shown in Additional File 1 Table S1, which was consistent to the finding delivered by categorical values. No effect of interaction between FBG and TG was observed after adjustment as shown in Additional file Table S2.

Table 3 shows similar results in subjects separated into men and women: (1) male: third and fourth quintiles of TyG (third quintile: OR 1.27, 95\% CI 1.02-1.59, $\mathrm{p}=0.0309$; fourth quintile: OR 1.39, 95\% CI 1.11-1.74, $\mathrm{p}=0.0042)$, HDL-C (OR 1.36, 95\% CI 1.16-1.58, $\mathrm{p}<0.0001)$ and $\mathrm{TG} \geq 1.7 \mathrm{mmol} / \mathrm{L}$ $(1.7 \leq \mathrm{TG}<2.3 \mathrm{mmol} / \mathrm{L}:$ OR 1.22 , $95 \%$ CI $1.02-1.47$, $\mathrm{p}=0.0336)$ are all associated with HTN. To be noted, a slight negative association between HbA1c and HTN was revealed in the male population (OR 0.76, 95\% CI 0.59-0.97, $\mathrm{p}=0.0292$ ). (2) female: fourth and fifth 

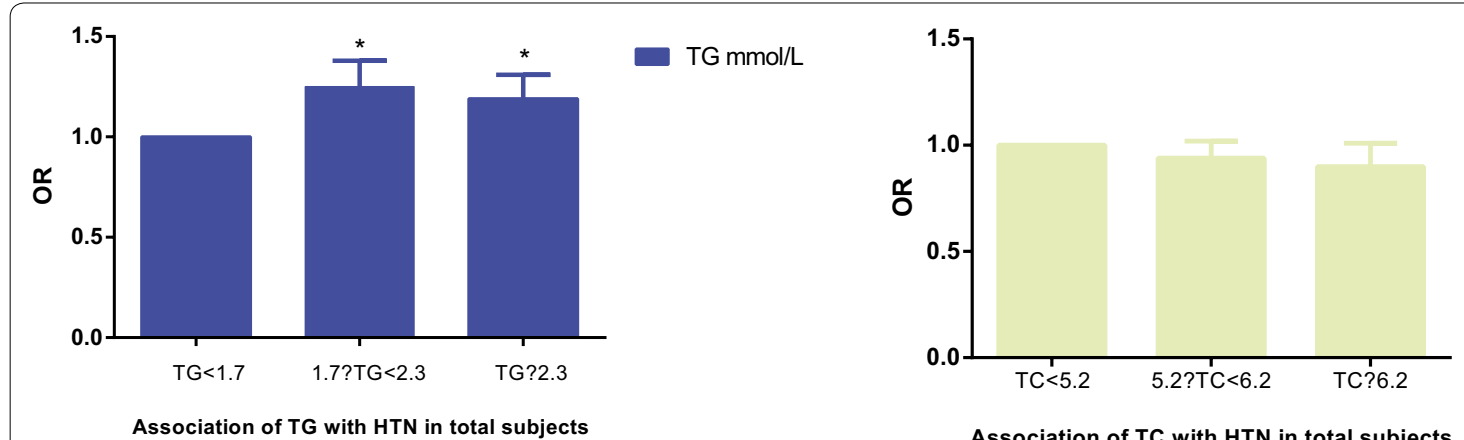

$\mathrm{TC} \mathrm{mmol} / \mathrm{L}$

Association of TC with HTN in total subjects

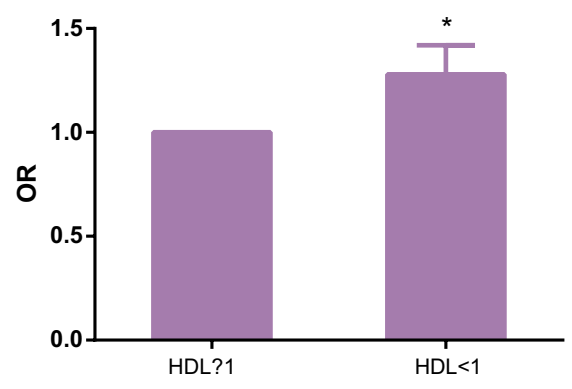

$\mathrm{HDL} \mathrm{mmol/L}$

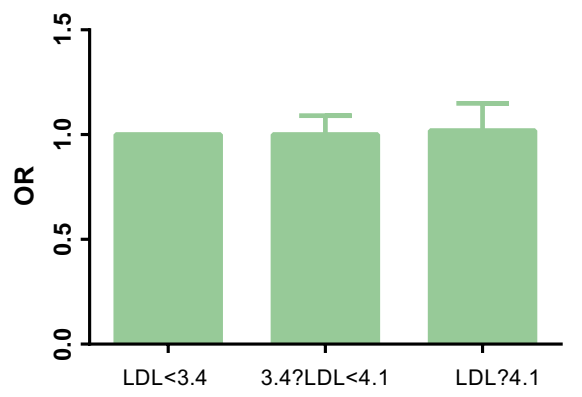

LDL mmol/L

Association of HDL with HTN in total subjects

Association of LDL with HTN in total subjects

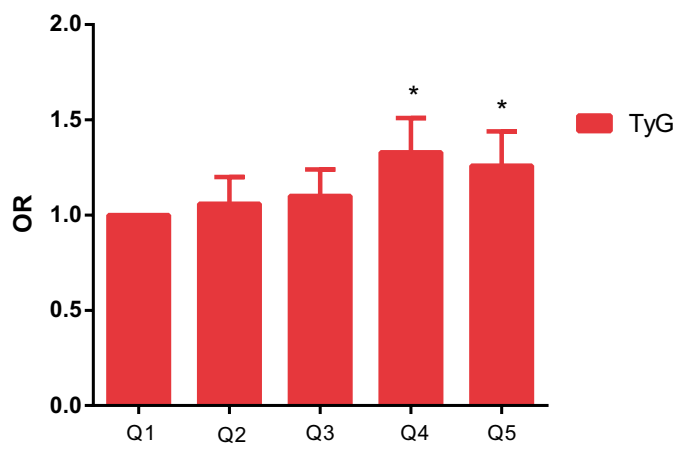

Association of TyG index with HTN in total subjects

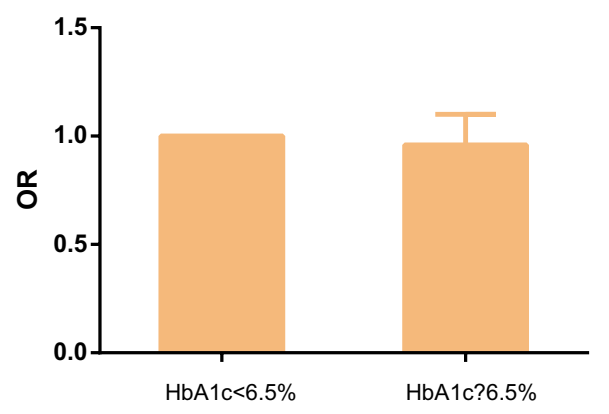

$\mathrm{HbA} 1 \mathrm{c} \%$

Association of HbA1c with HTN in total subjects

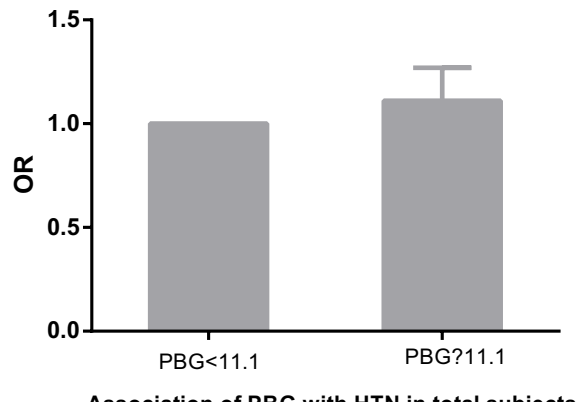

PBG $\mathrm{mmol} / \mathrm{L}$

Fig. 2 Association of the TyG index, glycemic, lipid parameters with HTN in total subjects in Model II (adjusted for age; center; history of CVDs; history of T2DM; hypoglycemic drugs; SBP; DBP; BMI; ALT; AST; WHR; eGFR; smoking habits; drinking habits. $P<0.05^{*}$ ) 
Table 3 Association of the TyG index, glycemic, lipid parameters with HTN by sex in Model 2

\begin{tabular}{|c|c|c|c|c|}
\hline \multirow[t]{2}{*}{ Variable } & \multicolumn{2}{|l|}{ Male } & \multicolumn{2}{|l|}{ Female } \\
\hline & OR $(95 \% \mathrm{Cl})$ & P-value & OR $(95 \% \mathrm{Cl})$ & P-value \\
\hline \multicolumn{5}{|l|}{$\mathrm{TG}, \mathrm{mmol} / \mathrm{L}$} \\
\hline$<1.7$ & 1.0 & & 1.0 & \\
\hline$\geq 1.7,<2.3$ & $1.22(1.02,1.47)$ & 0.0336 & $1.24(1.10,1.40)$ & 0.0003 \\
\hline$\geq 2.3$ & $1.14(0.96,1.36)$ & 0.1377 & $1.18(1.04,1.33)$ & 0.0098 \\
\hline \multicolumn{5}{|l|}{$\mathrm{TC}, \mathrm{mmol} / \mathrm{L}$} \\
\hline$<5.2$ & 1.0 & & 1.0 & \\
\hline$\geq 5.2,<6.2$ & $0.89(0.76,1.04)$ & 0.1454 & $0.95(0.86,1.06)$ & 0.3689 \\
\hline$\geq 6.2$ & $0.98(0.78,1.24)$ & 0.8749 & $0.87(0.77,0.99)$ & 0.0289 \\
\hline \multicolumn{5}{|c|}{$\mathrm{HDL}-\mathrm{C}, \mathrm{mmol} / \mathrm{L}$} \\
\hline$\geq 1$ & 1.0 & & 1.0 & \\
\hline$<1$ & $1.36(1.16,1.58)$ & $<0.0001$ & $1.20(1.04,1.39)$ & 0.0128 \\
\hline \multicolumn{5}{|c|}{$\mathrm{LDL}-\mathrm{C}, \mathrm{mmol} / \mathrm{L}$} \\
\hline$<3.4$ & 1.0 & & 1.0 & \\
\hline$\geq 3.4,<4.1$ & $0.99(0.83,1.18)$ & 0.9088 & $1.00(0.89,1.11)$ & 0.9433 \\
\hline$\geq 4.1$ & $1.15(0.89,1.48)$ & 0.2941 & $0.97(0.84,1.11)$ & 0.6651 \\
\hline \multicolumn{5}{|c|}{ TYG In transform Q5 } \\
\hline Q1 & 1.0 & & 1.0 & \\
\hline Q2 & $1.05(0.85,1.32)$ & 0.6358 & $1.05(0.91,1.22)$ & 0.4763 \\
\hline Q3 & $1.27(1.02,1.59)$ & 0.0309 & $1.01(0.88,1.17)$ & 0.8498 \\
\hline Q4 & $1.39(1.11,1.74)$ & 0.0042 & $1.28(1.11,1.49)$ & 0.0010 \\
\hline Q5 & $1.19(0.95,1.51)$ & 0.1376 & $1.25(1.07,1.47)$ & 0.0057 \\
\hline \multicolumn{5}{|l|}{$\mathrm{HbA} 1 \mathrm{c}, \%$} \\
\hline$<6.5$ & 1.0 & & 1.0 & \\
\hline$\geq 6.5$ & $0.76(0.59,0.97)$ & 0.0292 & $1.05(0.89,1.24)$ & 0.5480 \\
\hline \multicolumn{5}{|l|}{$\mathrm{PBG}, \mathrm{mmol} / \mathrm{L}$} \\
\hline$<11.1$ & 1.0 & & 1.0 & \\
\hline$\geq 11.1$ & $0.97(0.77,1.22)$ & 0.7897 & $1.17(0.98,1.40)$ & 0.0798 \\
\hline
\end{tabular}

Model 2: Adjusted for age; center; history of CVDs; history of T2DM; hypoglycemic drugs; SBP; DBP; BMl; ALT; AST; WHR; eGFR; smoking habits; drinking habits

quintiles of TyG (fourth quintile: OR 1.28, 95\% CI 1.11$1.49, \mathrm{p}=0.0010$; fifth quintile: OR 1.25 , 95\% CI $1.07-$ $1.47, \mathrm{p}=0.0057)$, HDL-C (OR 1.20, 95\% CI 1.04-1.39, $\mathrm{p}<0.0001), \mathrm{TG} \geq 1.7 \mathrm{mmol} / \mathrm{L} \quad(1.7 \leq \mathrm{TG}<2.3:$ OR 1.24 , 95\% CI 1.10-1.40, $\mathrm{p}=0.0003$; TG $\geq 2.3 \mathrm{mmol} / \mathrm{L}:$ OR 1.18 , 95\% CI 1.04-1.33, $\mathrm{p}=0.0098)$ and PBG (OR 1.03, 95\% CI $1.01-1.05, \mathrm{p}=0.0023)$ are associated with HTN. The above results are displayed as a color-coded figure shown in Fig. 3.

\section{Associations between the TyG index and HTN in individuals with LDL-C $<2.6 \mathrm{mmol} / \mathrm{L}$ or $\mathrm{HDL}-\mathrm{C}>1.0 \mathrm{mmol} / \mathrm{L}$}

As the Chinese guideline for the management of dyslipidemia in adults (revised in 2016) [31] suggests, the population was categorized into two groups, $\mathrm{LDL}-\mathrm{C}<2.6 \mathrm{mmol} / \mathrm{L}$ (ideal value) and
HDL-C $\geq 1.0 \mathrm{mmol} / \mathrm{L}$ (low risk value). As Table 4 shows, high TyG levels (the fourth and fifth quantile) were still significantly associated with HTN even when LDL-C or HDL-C was well-controlled (When LDL-C was wellcontrolled: the forth quintile of TyG: OR 1.26, 95\% CI $1.10-1.44, \mathrm{p}=0.0026$; the fifth quintile of TyG: OR 1.24, 95\% CI 1.07-1.44, $\mathrm{p}=0.0080$. When HDL-C was wellcontrolled: the forth quintile of TyG: OR $1.28,95 \% \mathrm{CI}$ 1.09-1.50, $\mathrm{p}=0.0007$; the fifth quintile of TyG: OR 1.27, 95\% CI 1.06-1.51, $\mathrm{p}=0.0026)$. Moreover, medium and high TG levels $(\geq 1.7 \mathrm{mmol} / \mathrm{L})$ as well as high HDL-C levels $(\geq 1 \mathrm{mmol} / \mathrm{L})$ also remained associated with HTN when LDL-C or HDL-C was well-controlled. The associations were not statistically significant in PBG, HbA1c or other lipid parameters.

\section{Associations between the TyG index and HTN for stratified subgroups of age, BMI and eGFR}

Stratified analyses were conducted in the different subgroups to further validate the abovementioned results, shown in Table 5. The present study suggested that compared with participants with lower TyG levels, subjects with higher TyG levels (the fourth and fifth quintile) were more closely associated with HTN in the older age ( $\geq 55$ years), higher level of BMI $\left(\geq 24 \mathrm{~kg} / \mathrm{m}^{2}\right)$ and both eGFR subgroups. To be noted, these associations were most significant in the subjects that were both in the subgroup of the forth quintile of TyG and the subgroup of oldest age ( $\geq 65$ years) (OR 1.67, 95\% CI 1.30$2.14, \mathrm{p}<0.0001)$, obesity $\left(\mathrm{BMI} \geq 28 \mathrm{~kg} / \mathrm{m}^{2}\right) \quad(\mathrm{OR} 1.85$, 95\% CI 1.29-2.66, $\mathrm{p}=0.0009)$ or lower eGFR $(<90 \mathrm{~mL} /$ $\left.\left(\mathrm{min} \cdot 1.73 \mathrm{~m}^{2}\right)\right)($ OR 1.72, 95\% CI 1.33-2.21, $\mathrm{p}<0.0001)$.

Our study also shows that people with borderline high TG levels $(\geq 1.7,<2.3 \mathrm{mmol} / \mathrm{L})$ were associated with HTN either in all age subgroups, normal and overweight subgroups (BMI $\left.\geq 18.5 \mathrm{~kg} / \mathrm{m}^{2},<28 \mathrm{~kg} / \mathrm{m}^{2}\right)$ or both eGFR subgroups. Meanwhile, people with high TG levels $(\geq 2.3 \mathrm{mmol} / \mathrm{L})$ were associated with HTN either in the medium age ( $\geq 55,<65$ years) subgroup, overweight and obesity subgroups (BMI $\geq 28 \mathrm{~kg} / \mathrm{m}^{2}$ ) or both eGFR subgroups. People with lower HDL-C levels $(<1 \mathrm{mmol} / \mathrm{L})$ were associated with HTN either in younger and medium age subgroups ( $<65$ years), normal and overweight subgroups $\left(B M I \geq 18.5 \mathrm{~kg} / \mathrm{m}^{2},<28 \mathrm{~kg} / \mathrm{m}^{2}\right.$ ) or the higher eGFR $\left(\geq 90 \mathrm{~mL} /\left(\min \cdot 1.73 \mathrm{~m}^{2}\right)\right)$ subgroup. The TC $(\geq 6.2 \mathrm{mmol} / \mathrm{L})$ subgroup was associated with HTN only in the medium age subgroup ( $\geq 55,<65$ years) or with the higher eGFR $\left(\geq 90 \mathrm{~mL} /\left(\mathrm{min} \cdot 1.73 \mathrm{~m}^{2}\right)\right)$ subgroup. A slight association between PBG and HTN was observed only in medium age, overweight and higher eGFR subgroups. However, no apparent association was observed in HbA1c stratified subgroups. The interaction between TG and BMI $(p=0.0339)$ and between HDL-C and age 

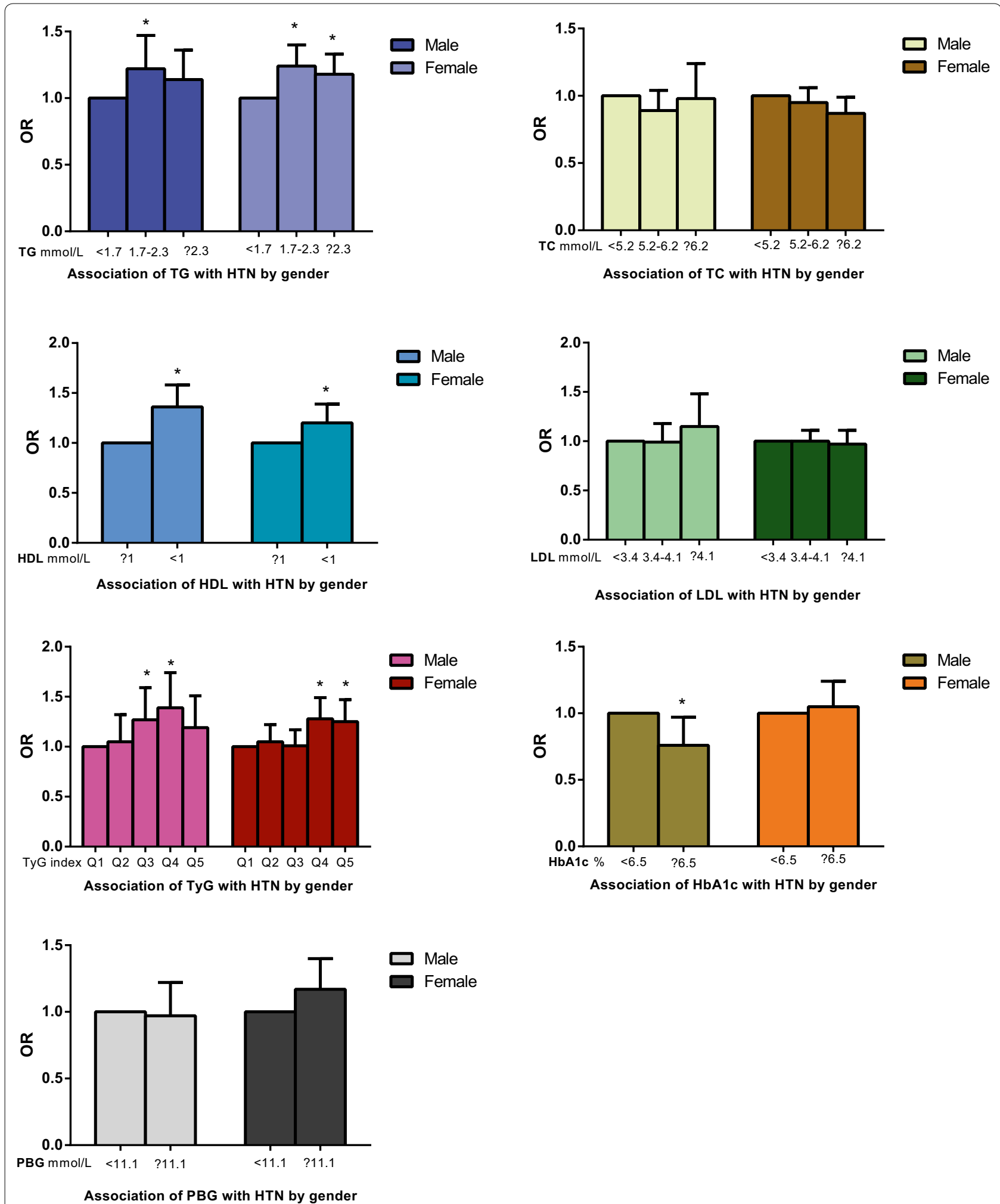

Fig. 3 Association of the TyG index, glycemic, lipid parameters with HTN by gender in Model II (adjusted for age; center; history of CVDs; history of T2DM; hypoglycemic drugs; SBP; DBP; BMI; ALT; AST; WHR; eGFR; smoking habits; drinking habits. $\mathrm{P}<0.05^{*}$ ) 
Table 4 Associations between the TyG index and HTN in people with $\mathrm{LDL}-\mathrm{C}<2.6 \mathrm{mmol} / \mathrm{L}$ or $\mathrm{HDL}-\mathrm{C}>1.0 \mathrm{mmol} / \mathrm{L}$

\begin{tabular}{|c|c|c|c|c|}
\hline \multirow[t]{2}{*}{ Variable } & \multicolumn{2}{|c|}{$\mathrm{HDL}-\mathrm{C}>1.0 \mathrm{mmol} / \mathrm{L}$} & \multicolumn{2}{|c|}{$\mathrm{LDL}-\mathrm{C}<2.6 \mathrm{mmol} / \mathrm{L}$} \\
\hline & OR $(95 \% \mathrm{CI})$ & P-value & OR $(95 \% \mathrm{Cl})$ & P-value \\
\hline \multicolumn{5}{|l|}{$\mathrm{TG}, \mathrm{mmol} / \mathrm{L}$} \\
\hline$<1.7$ & 1.0 & & 1.0 & \\
\hline$\geq 1.7,<2.3$ & $1.24(1.11,1.39)$ & 0.0001 & $1.19(1.06,1.34)$ & 0.0040 \\
\hline$\geq 2.3$ & $1.19(1.05,1.33)$ & 0.0048 & $1.12(0.99,1.28)$ & 0.0772 \\
\hline \multicolumn{5}{|l|}{$\mathrm{TC}, \mathrm{mmol} / \mathrm{L}$} \\
\hline$<5.2$ & 1.0 & & 1.0 & \\
\hline$\geq 5.2,<6.2$ & $0.97(0.88,1.06)$ & 0.4546 & $0.99(0.89,1.10)$ & 0.8933 \\
\hline$\geq 6.2$ & $0.94(0.83,1.05)$ & 0.2736 & $0.96(0.85,1.09)$ & 0.5788 \\
\hline \multicolumn{5}{|c|}{$\mathrm{HDL}-\mathrm{C}, \mathrm{mmol} / \mathrm{L}$} \\
\hline$\geq 1$ & - & & 1.0 & \\
\hline$<1$ & - & & $1.26(1.05,1.50)$ & 0.0125 \\
\hline \multicolumn{5}{|c|}{ LDL-C, mmol/L } \\
\hline$<3.4$ & 1.0 & & - & \\
\hline$\geq 3.4,<4.1$ & $1.00(0.90,1.11)$ & 0.9993 & - & \\
\hline$\geq 4.1$ & $1.04(0.92,1.18)$ & 0.5327 & - & \\
\hline \multicolumn{5}{|c|}{ TYG In transform Q5 } \\
\hline Q1 & 1.0 & & 1.0 & \\
\hline Q2 & $1.03(0.90,1.17)$ & 0.6633 & $1.09(0.93,1.29)$ & 0.2728 \\
\hline Q3 & $1.05(0.92,1.20)$ & 0.4438 & $1.12(0.95,1.31)$ & 0.1734 \\
\hline Q4 & $1.26(1.10,1.44)$ & 0.0007 & $1.28(1.09,1.50)$ & 0.0026 \\
\hline Q5 & $1.24(1.07,1.44)$ & 0.0042 & $1.27(1.06,1.51)$ & 0.0080 \\
\hline \multicolumn{5}{|l|}{$\mathrm{HbA} 1 \mathrm{c}, \%$} \\
\hline$<6.5$ & 1.0 & & 1.0 & \\
\hline$\geq 6.5$ & $1.01(0.87,1.18)$ & 0.8694 & $0.96(0.78,1.20)$ & 0.7372 \\
\hline \multicolumn{5}{|l|}{$\mathrm{PBG}, \mathrm{mmol} / \mathrm{L}$} \\
\hline$<11.1$ & 1.0 & & 1.0 & \\
\hline$\geq 11.1$ & $1.13(0.97,1.31)$ & 0.1196 & $1.22(0.98,1.50)$ & 0.0690 \\
\hline
\end{tabular}

Adjusted for age; center; sex; history of CVDs; history of T2DM; hypoglycemic drugs; SBP; DBP; BMI; ALT; AST; WHR; eGFR; smoking habits; drinking habits

$(\mathrm{p}=0.0368)$ was significant after adjusting for potential confounders, indicating there would be excess risk due to the additive interaction. When comparing the OR of TyG, lipid and glycemic parameters, the OR of TyG stands out the most, indicating that TyG can be a better discriminator of HTN.

\section{Discussion}

\section{Main findings}

As far as we know, this is the first study to investigate the associations of the TyG index, glycemic, lipid parameters with HTN in a Chinese general population with large sample, multicenter survey. The following are the main findings of this study: (1) The TyG index is significantly associated with HTN and remains significant after LDL-C or HDL-C was well-controlled, and the association of the TyG index with HTN is stronger than lipid or glycemic parameters. (2) HDL-C, TG and PBG are also associated with HTN but are inferior to the TyG index. (3) Further stratification shows that people with a larger BMI $\left(\geq 24 \mathrm{~kg} / \mathrm{m}^{2}\right)$, older age $(\geq 65)$ and lower eGFR $\left(<90 \mathrm{~mL} /\left(\min \cdot 1.73 \mathrm{~m}^{2}\right)\right)$ have higher risks of HTN when the TyG index is at a high level (in the fourth and fifth quintiles). Therefore the TyG index is a better discriminator for the risk of HTN compared with lipid and glycemic parameters.

\section{Glycemic parameters and HTN}

It was shown in previous studies that patients with CVDs can benefit from better glycemic control [36, 37]. This research has placed its focus on the average levels and ideal targets of FPG and HbA1c, for the most part. However, this study found only a slight association between HTN and PBG and no apparent association between HTN and HbA1c levels, even in subjects with older age, larger BMI and lower eGFR. Moreover, a slight negative association between HbA1c and HTN was revealed in the male population. There are potential limitations that exist in the assessment of these two glycemic parameters. Although elevated glucose concentration has been treated as a regulable cardiovascular risk factor and a more robust predictor of diabetes than the TyG index [38], FPG only serves as a less effective predictor of cardiovascular outcomes [39]. HbA1c is recommended as the most reliable parameter in the short-term evaluation of glycemic control, but substantial differences have been uncovered between HbA1c and average glycemic level. Especially, similar average glycemic levels could yield considerable discrepancies in HbA1c levels because glucose metabolism and hemoglobin glycation rate might vary corresponding among different individuals $[40,41]$.

\section{Lipid parameters and HTN}

Dyslipidemia remains as a conventional risk factor for CVD including atherosclerosis, particularly in the general population. Little research has been carried out on the association between lipid parameters and HTN. A 6.4-year follow-up study of 5971 middle-eastern women reported that the predictive value between TG, HDL-C, TG/HDL-C and HTN was most significant among several lipid parameters [42]. Studies on adolescents also reached similar conclusions [43-45]. The results of our study, which show an association between TG, HDL-C and HTN, agree with earlier ones. The sex-specific factors, such as hormone levels, might account for the discrepancies between TG and HTN in different sexes. Reports are limited about the interaction between BMI and TG or HDL-C and age on blood pressure. It is documented that body weight and height decrease and fat 


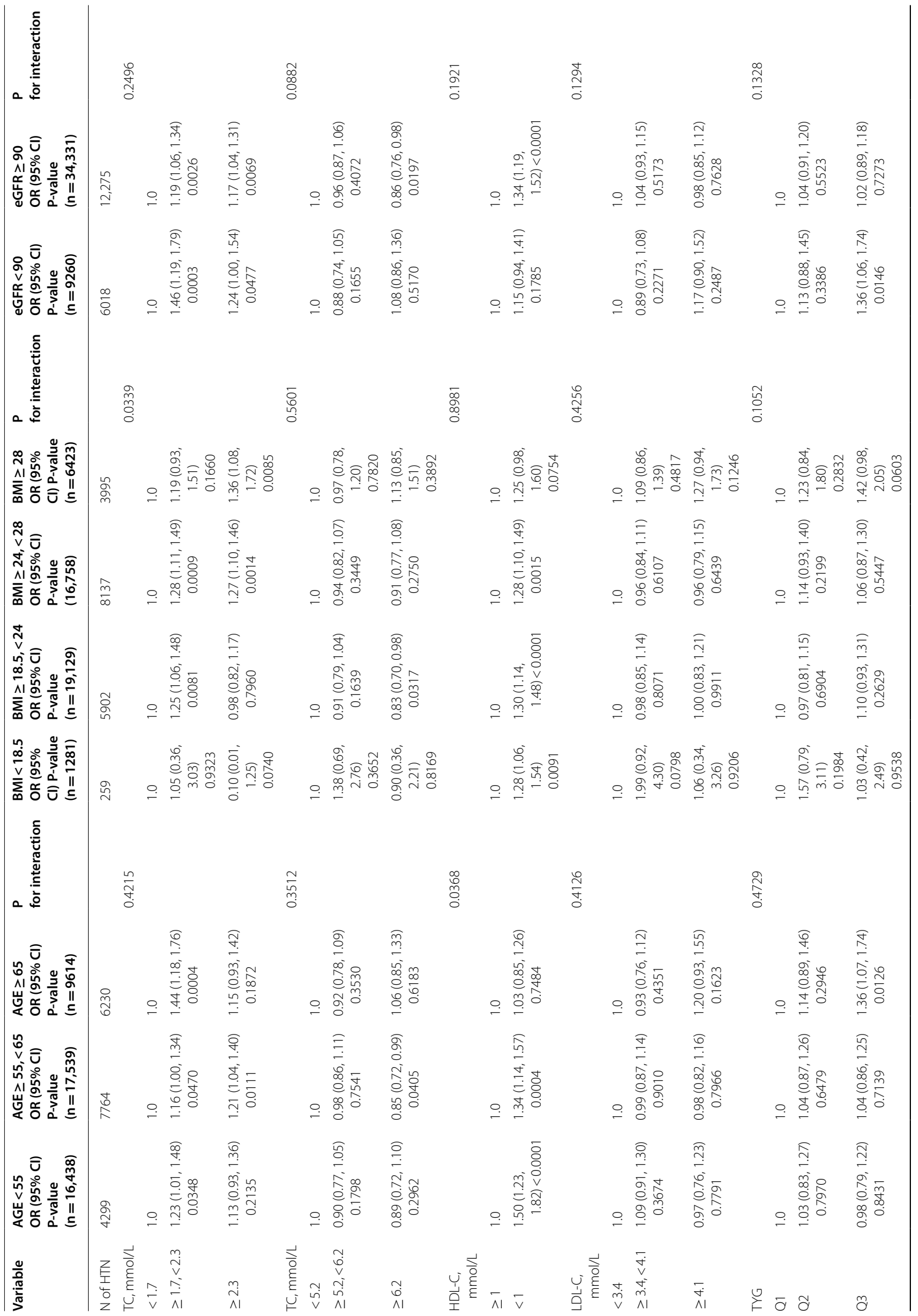




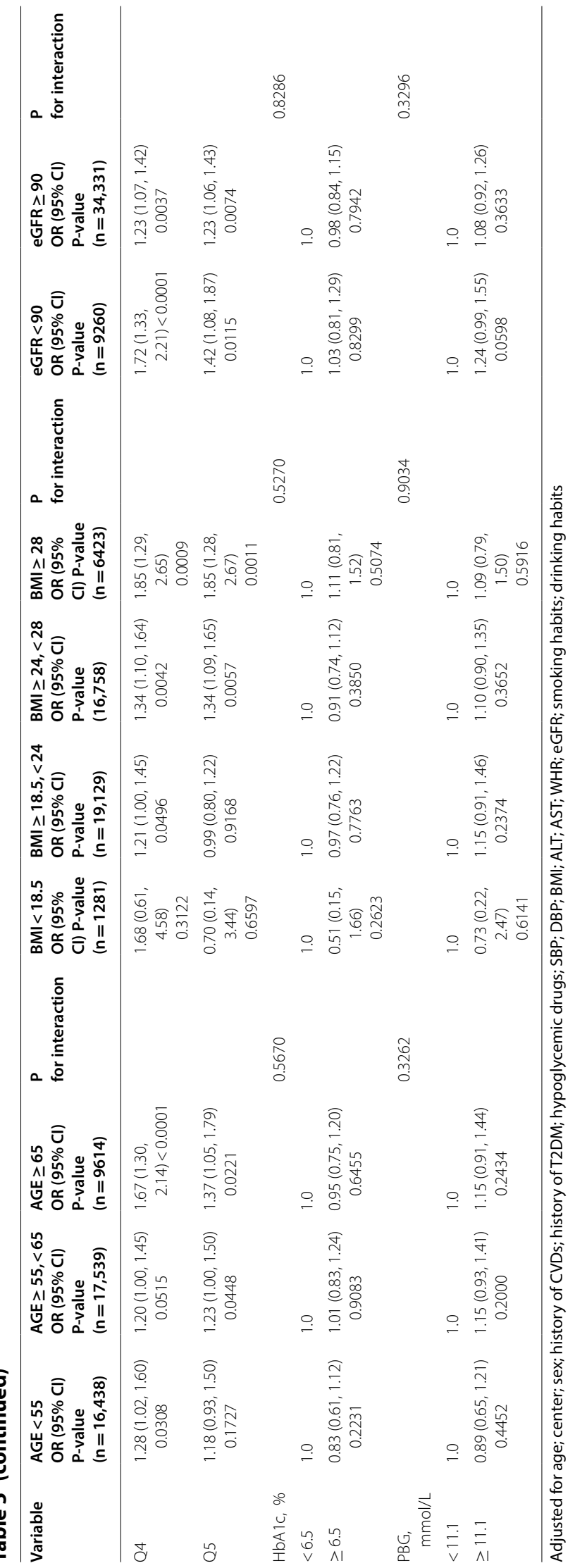


mass increase, trunk, and visceral adipose tissue redistribute across higher age [46, 47]. There is a review speculating that increased adipose tissue, which closely relates to TG, contributes to obesity and thus generates an environment in which hypertension can develop [48]. It might account for the interaction between HDL-C and age that low HDL-C is more prevalent in younger and middle age groups; also, longer exposure to environmental and genetic factors might influence the results [49]. Although ACC/AHA and ESC/EAS guidelines have recommended LDL-C to be the most crucial lipid risk factor and therapeutic goal for CVDs [50], LDL-C is not effectively indicative in our study. Moreover, Assmann et al. have proven that the number of clinical events is still alarming regardless of currently desirable LDL-C lowering therapies $[51,52]$. In fact, even if LDL-C or HDL-C is well-controlled, a higher TyG index or hypertriglyceridemia is still significantly associated with HTN in our study.

\section{The mechanisms between the TyG index and HTN}

Although the relevant pathophysiological mechanisms responsible for the association between the TyG index and HTN is unclear, several studies suggested the possible mechanism by which IR might affect elevating blood pressure. Theoretically, IR is an essential pathological element involved in metabolic syndrome and a risk factor for elevated blood pressure. Numerous studies have suggested that excess visceral fat represents the cause of metabolic abnormalities leading to increased IR and cardiometabolic risk, including the risk of HTN $[53,54]$. Therefore the measurement of IR is valuable in indicating HTN development [53, 54]. In recent years, it has been revealed that the TyG index was closely associated with IR and recommended as a surrogate index of IR [20,55,56]. Several studies lend support to the clinical significance of the TyG index for the assessment of atherosclerosis and vascular damage $[57,58]$. It has been also reported that the TyG index could be an efficient marker to indicate ischemic stroke, symptomatic CADs and major adverse cardiovascular and cerebral events (MACCEs) [54, 59, 60]. Our findings show that the TyG index, an emerging measurable substitution of IR, is independently associated with HTN after various confounding factors adjustment, which are consistent with previous studies. We have also conducted analysis on other IR markers as shown in Additional file Table S3, demonstrating that the TyG index is a superior discriminator of HTN than other IR markers. IR-compensatory hyperinsulinemia can generate overactivation of the carotid body, bringing on an escalation in sympathetic nervous system activity, further prompting adrenaline and norepinephrine's secretion, and eventually resulting in cardiac output increases and peripheral vascular resistance [61]. The vascular smooth muscle may be thickened in high concentrated catecholamine, inducing HTN development [62]. Moreover, blood pressure can also be elevated by IR through the activation of the renin-angiotensinaldosterone system and the increase in endothelin synthesis $[63,64]$. Such an increase may contract the blood vessels, decrease the prostacyclin (PGI2) and prostaglandin E2 (PGE2) circulated in vessels which are supposed to be dilated by them $[19,65]$, and finally proliferate in the vascular smooth muscle, contributing to HTN development.

To be noted, higher eGFR has a close bond to hypertension in the present study. Although the underlying mechanism has not been fully understood, some evidence leads to regard hyperfiltration both a cause and a consequence [66]. Glomerular hyperfiltration can be caused by afferent arteriolar vasodilation, or by efferent arteriolar vasoconstriction owing to activation of the renin-angiotensin-aldosterone system, thus prompting to glomerular hypertension. Thus, early recognition of hyperfiltration will enable clinicians to detect renal diseases and prevent progression of hypertension.

Interestingly, in our study, it is first revealed that the TyG index might be superior to mere glycemic or lipid parameters in associating HTN development. The TyG index is applied in assessing the joint value of TG and FPG because the two parameters are intensively interrelated. Hypertriglyceridemia remains one of the most prevalent abnormalities in T2DM patients, and its association with increased risk of CVDs has been fully demonstrated [67-69]. TG might prompt the formation of atherosclerotic plaque, while glycemic level might be involved in endothelial cells and platelet dysfunction [70-73]. Their values in association with HTN might be better interpreted when they are considered as a whole. Our study shows that the TyG index helps to identify potential risks in individuals who would otherwise be neglected. Clinicians usually put their focus merely on individuals with high FBG or TG. Such conventional clinical practice might possibly miss out on some of the potential risk groups whose FBG and TG are in the normal or borderline ranges.

\section{Limitations}

Our seven-region community-based samples, which representatively demonstrate the distribution of different regions in China, largely and positively influence the research. However, there are still limitations in our 
study. First, we were not able to directly measure IR in our study population and further compare the surrogate indices with direct markers of IR. Second, because the data of this research was from Chinese elderly individuals, it remains uncertain whether the findings can be applicable to other ethnic groups. Finally, as a feature of the cross-sectional study, only associations, rather than causality, can be established. More prospective studies are needed to identify causal relationship between the TyG index and HTN.

\section{Conclusion}

To conclude, the results of our study reveal a significant association between the TyG index and HTN in Chinese elderly individuals; and it is superior to other lipid profiles and HbA1c and PBG. Therefore we propose that the TyG index could be a more efficient, useful and simple index for the screening and managing of HTN (Additional file 1).

\section{Supplementary information}

Supplementary information accompanies this paper at https://doi. org/10.1186/s12933-020-01077-6.

Additional file 1: Table S1. Association of the TyG index, glycemic, lipid parameters as continuous variables with HTN in total subjects. Table S2. Interaction of FBG with TG on hypertension. Table S3. Association of TG/ HDL and HOMA-IR with HTN in total subjects.

\section{Abbreviations}

ALT: Alanine transferase; AST: Aspartate transferase; BMI: Body mass index; CHD: Coronary heart disease; CVD: Cardiovascular disease; Cl: Confidence interval; DBP: Diastolic blood pressure; eGFR: Estimated glomerular filtration rate; FBG: Fasting plasma glucose; GGT: Gamma-glutamyl transferase; HbA1c: Glycosylated hemoglobin; HDL-C: High-density lipoprotein cholesterol; HR: Heart rate; HTN: Hypertension; IR: Insulin resistance; LDL-C: Low-density lipoprotein cholesterol; MACCEs: Major adverse cardiovascular and cerebral events; OGTT: Glucose tolerance test; OR: Odds ratio; PBG: 2 h post-load blood glucose; PGE2: Prostaglandin E2; PGI2: Prostacyclin; SBP: Systolic blood pressure; TC: High cholesterol; TG: Triglyceride; TyG: Triglyceride-glucose; T2DM: Type 2 diabetes; WHR: Waist circumference/hip circumference.

\section{Acknowledgements}

We would like to thank the participants in this study.

\section{Authors' contributions}

YM, JW and BZ contributed to the conception and design of the study. $\mathrm{KC}, \mathrm{WY}$, AW, WW, ZG, XT, LY, QW, ZL, GQ and LC recruited the subjects and supervised the study. JW and BZ analyzed the data. BZ wrote the initial draft of the paper. BZ and JW contributed equally to this work. YM, JW and BZ contributed to the writing, reviewing, and revising of the manuscript. All authors read and approved the final manuscript.

\section{Funding}

The study is supported by the Chinese Society of Endocrinology, the Key Laboratory for Endocrine and Metabolic Diseases of Ministry of Health (1994DP131044), the National Key New Drug Creation and Manufacturing Program of Ministry of Science and Technology (2012ZX09303006-001), the National High Technology Research and Development Program of China (863 Program, 2011AA020107), National Science Foundation of China (81300717) National Science and Technology Major Project 288 (2011ZX09307-001-08), the REACTION Study.

\section{Data availability}

The datasets used to support this study are not freely available due to participants' privacy protection.

\section{Ethics approval and consent to participate}

The study protocol was approved by the Committee on Human Research at Rui-Jin Hospital affiliated with the School of Medicine, Shanghai Jiao Tong University. Written informed consents were obtained from all participants before data collection.

\section{Consent for publication}

Not applicable.

\section{Competing interests}

The authors declare that they have no competing interests.

\section{Author details}

${ }^{1}$ Medicine School of Nankai University, Tianjin, China. ${ }^{2}$ Department of Endocrinology, Chinese PLA General Hospital, 28 Fu Xing Road, Beijing 100853, People's Republic of China. ${ }^{3}$ Shanghai National Research Centre for Endocrine and Metabolic Diseases, State Key Laboratory of Medical Genomics, Shanghai Institute for Endocrine and Metabolic Diseases, Ruijin Hospital, Shanghai Jiaotong University School of Medicine, Shanghai, China. ${ }^{4}$ Dalian Central Hospital, Dalian, Liaoning, China. ${ }^{5}$ First Hospital of Lanzhou University, Lanzhou, Gansu, China. ${ }^{6}$ Zhongshan University Sun Yat-sen Memorial Hospital, Guangzhou, Guangdong, China. ${ }^{7}$ Southwest Medical University Affiliated Hospital, Luzhou, Sichuan, China. ${ }^{8}$ First Affiliated Hospital of Guangxi Medical University, Nanning, Guangxi, China. ${ }^{9}$ First Affiliated Hospital of Zhengzhou University, Zhengzhou, Henan, China. ${ }^{10}$ Wuhan Union Hospital, Huazhong University of Science and Technology, Wuhan, Hubei, China.

Received: 28 March 2020 Accepted: 2 July 2020

Published online: 14 July 2020

\section{References}

1. World Health Organization. World Health Statistics 2014. Geneva: World Health Organization; 2014. p. 175.

2. He J, Gu D, Wu X, et al. Major causes of death among men and women in China. N Engl J Med. 2005;353:1124-34.

3. He J, Gu D, Chen J, et al. Premature deaths attributable to blood pressure in China: a prospective cohort study. Lancet. 2009;374:1765-72.

4. O'Meara JG, Kardia SR, Armon JJ, Brown C, Boerwinkle E, Turner ST. Ethnic and sex differences in the prevalence, treatment, and control of dyslipidemia among hypertensive adults in the GENOA study. Arch Intern Med. 2004;164:1313-8

5. Laaksonen DE, Niskanen L, Nyyssönen K, Lakka TA, Laukkanen JA, Salonen JT. Dyslipidaemia as a predictor of hypertension in middle-aged men. Eur Heart J. 2008;29(20):2561-8. https://doi.org/10.1093/eurheartj/ehn061.

6. Sesso HD, Buring JE, Chown MJ, Ridker PM, Gaziano JM. A prospective study of plasma lipid levels and hypertension in women. Arch Intern Med. 2005;165(20):2420-7. https://doi.org/10.1001/archinte.165.20.2420.

7. Halperin RO, Sesso HD, Ma J, Buring JE, Stampfer MJ, Gaziano JM. Dyslipidemia and the risk of incident hypertension in men. Hypertension. 2006:47(1):45-50. https://doi.org/10.1161/01.hyp.0000196306.42418.0e.

8. Memon AR, Shaikh F, Memon ZA, Majeed Adnan BA. Assessment of lipid profile and serum total protien in patients of hypertension. Ann Pak Inst Med Sci. 2017;13(2):246-8.

9. Nordestgaard BG, Varbo A. Triglycerides and cardiovascular disease. Lancet. 2014;384(9943):626-35.

10. Ferrannini E, Cushman WC. Diabetes and hypertension: the bad companions. Lancet. 2012;380:601.

11. Janghorbani M, Bonnet F, Amini M. Glucose and the risk of hypertension in first-degree relatives of patients with type 2 diabetes. Hypertension Res. 2015;38(5):349-54. https://doi.org/10.1038/hr.2015.10.

12. Zinman B, Wanner C, Lachin JM, Fitchett D, Bluhmki E, Hantel S, EMPA-REG OUTCOME Investigators, et al. Empagliflozin, cardiovascular outcomes, and mortality in type 2 diabetes. N Engl J Med. 2015;373:2117-28.

13. Marso SP, Daniels GH, Brown-Frandsen K, Kristensen P, Mann JF, Nauck MA, LEADER Steering Committee; LEADER Trial Investigators, et al. 
Liraglutide and cardiovascular outcomes in type 2 diabetes. N Engl J Med. 2016;375:311-22.

14. Landsberg L, Aronne LJ, Beilin L, et al. Obesity-related hypertension: pathogenesis, cardiovascular risk, and treatment-a position paper of the obesity society and the American society of hypertension. Obesity. 2013;21:8-24.

15. Wang F, Han L, Hu D. Fasting insulin, insulin resistance and risk of hypertension in the general population: a meta-analysis. Clin Chim Acta. 2017:464:57-63.

16. De Fronzo RA, Tobin JD, Andres R. Glucose clamp technique: a method for quantifying insulin secretion and resistance. Am J Physiol. 1979;237(3):E214-23 PMID: 382871.

17. Singh B, Saxena A. Surrogate markers of insulin resistance: a review. World J Diabetes. 2010;1 (2):36-47.

18. Du T, Yuan G, Zhang M, Zhou X, Sun X, Yu X. Clinical usefulness of lipid ratios, visceral adiposity indicators, and the triglycerides and glucose index as risk markers of insulin resistance. Cardiovasc Diabetol. 2014;13:146.

19. Navarro-Gonzalez D, Sanchez-Inigo L, Pastrana-Delgado J, FernandezMontero A, Martinez JA. Triglyceride-glucose index (TyG index) in comparison with fasting plasma glucose improved diabetes prediction in patients with normal fasting glucose: the Vascular Metabolic CUN cohort. Prev Med. 2016;86:99-105.

20. Simental-Mendia LE, Rodriguez-Moran M, Guerrero-Romero F. The product of fasting glucose and triglycerides as surrogate for identifying insulin resistance in apparently healthy subjects. Metab Syndr Relat Disord. 2008;6:299-304.

21. Vasques AC, Novaes FS, de Oliveira Mda S, Souza JR, Yamanaka A, Pareja $J C$, et al. TyG index performs better than HOMA in a Brazilian population: a hyperglycemic clamp validated study. Diabetes Res Clin Pract. 2011;93(3):e98-100. https://doi.org/10.1016/.diabres.2011.05.030.

22. Jian S, Su-Mei N, Xue C, Jie Z, Xue-sen W. Association and interaction between triglyceride-glucose index and obesity on risk of hypertension in middle-aged and elderly adults. Clin Exp Hypertens. 2017;39:732-9.

23. Lee SB, Ahn CW, Lee BK, Kang S, Nam JS, You JH, et al. Association between triglyceride glucose index and arterial stiffness in Korean adults. Cardiovasc Diabetol. 2018;17:41. https://doi.org/10.1186/s1293 3-018-0692-1.

24. Lambrinoudaki I, Kazani MV, Armeni E, Georgiopoulos G, Tampakis K, Rizos D, et al. The TyG index as a marker of subclinical atherosclerosis and arterial stiffness in lean and overweight postmenopausal women. Hear Lung Circ. 2018;27:716-24.

25. Kim MK, Ahn CW, Kang S, Nam JS, Kim KR, Park JS. Relationship between the triglyceride glucose index and coronary artery calcification in Korean adults. Cardiovasc Diabetol. 2017;16:108. https://doi.org/10.1186/s1293 3-017-0589-4.

26. Mao Q, Zhou D, Li Y, Wang Y, Xu SC, Zhao XH. The triglyceride-glucose index predicts coronary artery disease severity and cardiovascular outcomes in patients with non-ST-segment elevation acute coronary syndrome. Dis Markers. 2019;2019:6891537.

27. Lee SH, Kwon HS, Park YM, Ha HS, Jeong SH, Yang HK, et al. Predicting the development of diabetes using the product of triglycerides and glucose: the Chungju Metabolic Disease Cohort (CMC) study. PLoS ONE. 2014;9:e90430.

28. Zhang M, Wang B, Liu Y, Sun X, Luo X, Wang C, et al. Cumulative increased risk of incident type 2 diabetes mellitus with increasing triglyceride glucose index in normal-weight people: the Rural Chinese Cohort Study. Cardiovasc Diabetol. 2017;16:30.

29. Lee DY, Lee ES, Kim JH, Park SE, Park CY, Oh KW, et al. Predictive value of triglyceride glucose index for the risk of incident diabetes: a 4-year retrospective longitudinal study. PLoS ONE. 2016;11:e0163465.

30. Ma YC, Zuo L, Chen JH, Luo Q, Yu XQ, Li Y, Xu JS, Huang SM, Wang LN, Huang $W$, et al. Modifed glomerular ftration rate estimating equation for Chinese patients with chronic kidney disease. J Am Soc Nephrol. 2006;17(10):2937-44.

31. Joint committee for guideline revision. 2016 Chinese guidelines for the management of dyslipidemia in adults. J Geriatr Cardiol. 2018;15(1):1-29. https://doi.org/10.11909/j.issn.1671-5411.2018.01.011.

32. International Expert Committee. International Expert Committee report on the role of the A1C assay in the diagnosis of diabetes. Diabetes Care. 2009;32:1327-34.
33. WHO Guidelines Approved by the Guidelines Review Committee. Use of Glycated Haemoglobin (HbA1c) in the Diagnosis of Diabetes Mellitus: Abbreviated Report of a WHO Consultation. Geneva; 2011.

34. Zhou B, Coorperative Meta-Analysis Group Of China Obesity Task Force. Zhonghua liu xing bing xue za zhi = Zhonghua liuxingbingxue zazhi. 2002;23(1):5-10.

35. Khwaja A. KDIGO clinical practice guidelines for acute kidney injury. Nephron Clin Pract. 2012;120(4):c179-84. https://doi.org/10.1159/00033 9789.

36. Tinsley $L$, Kupelian V, D'Eon SA, et al. Association of glycemic control with reduced risk for large-vessel disease after more than 50 years of type 1 diabetes. J Clin Endocrinol Metab. 2017;102(10):3704-11.

37. Paneni F, Lüscher TF. Cardiovascular protection in the treatment of type 2 diabetes: a review of clinical trial results across drug classes. Am J Cardiol. 2017;120(1S):S17-27.

38. Chamroonkiadtikun $\mathrm{P}$, et al. The triglyceride-glucose index, a predictor of type 2 diabetes development: a retrospective cohort study. Prim Care Diabetes. 2019. https://doi.org/10.1016/j.pcd.2019.08.004.

39. Borg R, Kuenen JC, Carstensen B, et al. $\mathrm{HbA}_{1}$ (c) and mean blood glucose show stronger associations with cardiovascular disease risk factors than do postprandial glycaemia or glucose variability in persons with diabetes: the A1C-Derived Average Glucose (ADAG) study. Diabetologia. 2011;54(1):69-72.

40. Cavagnolli G, Pimentel AL, Freitas PA, et al. Factors affecting A1C in non-diabetic individuals: review and meta-analysis. Clin Chim Acta. 2015;445:107-14.

41. McCarter RJ, Hempe JM, Gomez R, Chalew SA. Biological variation in $\mathrm{HbA1c}$ predicts risk of retinopathy and nephropathy in type 1 diabetes. Diabetes Care. 2004;27:1259-64.

42. Tohidi M, Hatami M, Hadaegh F, Azizi F. Triglycerides and triglycerides to high-density lipoprotein cholesterol ratio are strong predictors of incident hypertension in middle eastern women. J Hum Hypertens. 2012;26(9):525-32. https://doi.org/10.1038/jhh.2011.70.

43. Urbina EM, Khoury PR, McCoy CE, Dolan LM, Daniels SR, Kimball TR. Triglyceride to HDL-C ratio and increased arterial stiffness in children, adolescents, and young adults. Pediatrics. 2013;131(4):e1082-90. https:// doi.org/10.1542/peds.2012-1726.

44. Quijada Z, Paoli M, Zerpa Y, Camacho N, Cichetti R, Villarroel V, ArataBellabarba G, Lanes R. The triglyceride/HDL-cholesterol ratio as a marker of cardiovascular risk in obese children; association with traditional and emergent risk factors. Pediatr Diabetes. 2008;9(5):464-71. https://doi.org/ 10.1111/j.1399-5448.2008.00406.x.

45. Di Bonito P, Moio N, Scilla C, Cavuto L, Sibilio G, Sanguigno E, Forziato C, Saitta F, lardino MR, Di Carluccio C, Capaldo B. Usefulness of the high triglyceride-to-HDL cholesterol ratio to identify Cardiometabolic risk factors and preclinical signs of organ damage in outpatient children. Diabetes Care. 2012;35(1):158-62. https://doi.org/10.2337/dc11-1456.

46. Moon HK, Kim EG. Comparing validity of body mass index, waist to hip ratio, and waist circumference to cardiovascular disease risk factors in Korean elderly. Korean J Nutr. 2005;38:445-54.

47. Perissinotto E, Pisent C, Sergi G, Grigoletto F, ILSA Working Group (Italian Longitudinal Study on Ageing). Anthropometric measurements in the elderly: age and gender differences. Br J Nutr. 2002;87(2):177-86. https:// doi.org/10.1079/bjn2001487.

48. Bogaert YE, Linas $\mathrm{S}$. The role of obesity in the pathogenesis of hypertension, Nature clinical practice. Nephrology. 2009;5(2):101-11. https://doi. org/10.1038/ncpneph1022.

49. Gharipour M, Sadeghi M, Hosseini M, Andalib E, Boroujeni MB, Sarrafzadegan $\mathrm{N}$. Effect of age on the phenotype of metabolic syndrome in developing country. Adv Biomed Res. 2015;4:103. https://doi. org/10.4103/2277-9175.157796.

50. Catapano AL, Graham I, De Backer G, Wiklund O, Chapman MJ, Drexel H, Hoes AW, Jennings CS, Landmesser U, Pedersen TR, Reiner Ž. 2016 ESC/ EAS Guidelines for the Management of Dyslipidaemias. Atherosclerosis. 2016;253:281-344. https://doi.org/10.1016/j.atherosclerosis.2016.08.018.

51. Deedwania P, Barter P, Carmena R, Fruchart J-C, Grundy SM, Haffner S, Kastelein JJP, LaRosa JC, Schachner H, Shepherd J, et al. Reduction of lowdensity lipoprotein cholesterol in patients with coronary heart disease and metabolic syndrome: analysis of the Treating to New Targets study. Lancet. 2006;368(9539):919-28. 
52. Cholesterol Treatment Trialists C, Mihaylova B, Emberson J, Blackwell L, Keech A, Simes J, Barnes EH, Voysey M, Gray A, Collins R, et al. The effects of lowering LDL cholesterol with statin therapy in people at low risk of vascular disease: meta-analysis of individual data from 27 randomised trials. Lancet. 2012;380(9841):581-90.

53. Kotchen TA, Grim CE, Kotchen JM, et al. Altered relationship of blood pressure to adiposity in hypertension. Am J Hypertens. 2008;21:284-9.

54. Manrique C, Lastra G, Gardner M, et al. The renin angiotensin aldosterone system in hypertension: roles of insulin resistance and oxidative stress. Med Clin North Am. 2009;93:569-82.

55. Vasques AC, Novaes FS, de Oliveira MdaS, et al. TyG index performs better than HOMA in a Brazilian population: a hyperglycemic clamp validated study. Diabetes Res Clin Pract. 2011;93:e98-100.

56. Guerrero-Romero F, Villalobos-Molina R, Jimenez-Flores JR, et al. Fasting triglycerides ' and glucose index as a diagnostic test for insulin resistance in young adults. Arch Med Res. 2016;47:382-7.

57. da Silva A, Caldas A, Hermsdorff H, Bersch-Ferreira ÂC, Torreglosa CR, Weber B, Bressan J. Triglyceride-glucose index is associated with symptomatic coronary artery disease in patients in secondary care. Cardiovasc Diabetol. 2019;18(1):89. https://doi.org/10.1186/s12933-019-0893-2.

58. Zhao S, Yu S, Chi C, et al. Association between macro- and microvascular damage and the triglyceride glucose index in community-dwelling elderly individuals: the Northern Shanghai Study. Cardiovasc Diabetol. 2019;18:95. https://doi.org/10.1186/s12933-019-0898-X.

59. Shi W, Xing L, Jing L, Tian Y, Yan H, Sun Q, Dai D, Shi L, Liu S. Value of triglyceride-glucose index for the estimation of ischemic stroke risk: insights from a general population. Nutr Metab Cardiovasc Dis. 2020;30(2):245-53. https://doi.org/10.1016/j.numecd.2019.09.015.

60. Luo E, Wang D, Yan G, Qiao Y, Liu B, Hou J, Tang C. High triglycerideglucose index is associated with poor prognosis in patients with acute ST-elevation myocardial infarction after percutaneous coronary intervention. Cardiovasc Diabetol. 2019;18(1):150. https://doi.org/10.1186/s1293 3-019-0957-3.

61. Arauz-Pacheco C, Lender D, "PG, et al. Relationship between insulin sensitivity, hyperinsulinemia, and insulin-mediated sympathetic activation in normotensive and hypertensive subjects. Am J Hypertens. 1996;9(12 Pt 1):1172-8.

62. Takagi M, Tanaka Y, Yamasaki Y, et al. Responsiveness of insulininduced cardiac sympathetic nerve activation associates with blood pressure regulation in diabetics. Am J Physiol Endocrinol Metab. 2003;284(5):E1022-6. https://doi.org/10.1152/ajpendo.00169.2002.

63. Fonseca VA. Insulin resistance, diabetes, hypertension, and reninangiotensin system inhibition: reducing risk for cardiovascular disease. J Clin Hypertens. 2006;8(10):713-20.

64. Khalil RA. Modulators of the vascular endothelin receptor in blood pressure regulation and hypertension. Curr Mol Pharmacol. 2011;4(3):176-86.

65. Frank HJ, Levin ER, Hu RM, et al. Insulin stimulates endothelin binding and action on cultured vascular smooth muscle cells. Endocrinology. 1993;133(3):1092. https://doi.org/10.1210/endo.133.3.8365355.

66. Helal I, Fick-Brosnahan G, Reed-Gitomer B, et al. Glomerular hyperfiltration: definitions, mechanisms and clinical implications. Nat Rev Nephrol. 2012;8:293-300. https://doi.org/10.1038/nrneph.2012.19.

67. Schmidt MI, Duncan BB, Bang H, et al. Identifying individuals at high risk for diabetes: the Atherosclerosis Risk in Communities Study. Diabetes Care. 2005;28:2013-8.

68. Wilson PW, Meigs JB, Sullivan L, Fox CS, et al. Prediction of incident diabetes mellitus in middle-aged adults: the Framingham Offspring Study. Arch Intern Med. 2007;167:1068-74.

69. Ren Y, Ren Q, Lu J, Guo X, Huo X, Ji L, Yang X (2018) Low triglyceride as a marker for increased risk of cardiovascular diseases in patients with longterm type 2 diabetes: A cross-sectional survey in China. Diabetes Metab Res Rev. 34(2). https://doi.org/10.1002/dmrr.2960.

70. Budoff M. Triglycerides and triglyceride-rich lipoproteins in the causal pathway of cardiovascular disease. Am J Cardiol. 2016;118(1):138-45.

71. Tenenbaum A, Klempfner R, Fisman EZ. Hypertriglyceridemia: a too long unfairly neglected major cardiovascular risk factor. Cardiovasc Diabetol. 2014;13:159.

72. Farhangkhoee H, Khan ZA, Barbin Y, Chakrabarti S. Glucose-induced up-regulation of CD36 mediates oxidative stress and microvascular endothelial cell dysfunction. Diabetologia. 2005;48(7):1401-10.

73. Zhou SX, Huo DM, He XY, et al. High glucose/lysophosphatidylcholine levels stimulate extracellular matrix deposition in diabetic nephropathy via platelet-activating factor receptor. Mol Med Rep. 2018;17(2):2366-72.

\section{Publisher's Note}

Springer Nature remains neutral with regard to jurisdictional claims in published maps and institutional affiliations.
Ready to submit your research? Choose BMC and benefit from:

- fast, convenient online submission

- thorough peer review by experienced researchers in your field

- rapid publication on acceptance

- support for research data, including large and complex data types

- gold Open Access which fosters wider collaboration and increased citations

- maximum visibility for your research: over $100 \mathrm{M}$ website views per year

At BMC, research is always in progress.

Learn more biomedcentral.com/submissions 